\title{
RETRACTED ARTICLE: Assessment of Pedestrian Refuge Islands on Vehicle Speed Changes and Pedestrian Safety: Case Study in Tehran
}

\author{
Arash Sadrayi $^{1} \cdot$ Mahmoud Saffarzadeh $^{1} \cdot$ Amin Mirza Boroujerdian $^{1}$
}

Received: 20 October 2015 / Revised: 25 July 2016/Accepted: 28 December 2016/Published online: 29 June 2017

(C) Iran University of Science and Technology 2018

The authors have retracted this article (1) due to significant overlap with previously published articles by the same authors $(1,2)$. All authors agree to this retraction. The online version of this article contains the full text of the retracted article as electronic supplementary material. 1) Arash Sadrayi, Mahmoud Saffarzadeh, Amin Mirza Boroujerdian, Assessment of Pedestrian Refuge Islands on Vehicle Speed Changes and Pedestrian Safety: Case Study in Tehran, International Journal of Civil Engineering 2)
Arash Sadrayi and Amin Mirza Boroujerdian, Assessment of Pedestrian Refuge Islands on Vehicle Speed Changes and Pedestrian Safety: Case Study in Tehran, Journal of Traffick and Logistics 3) Arash Sadrayi; Mahmoud Saffarzadeh; Amin Mirza Boroujerdian, An Assessment of the Impact of Pedestrian Refuge Islands on Vehicle Speed Changes and Pedestrian Safety: Case Study in Tehran International Journal of Transportation Engineering.

Electronic supplementary material The online version of this article (https://doi.org/10.1007/s40999-017-0244-1) contains supplementary material, which is available to authorized users.

Mahmoud Saffarzadeh

saffar_m@modares.ac.ir

Arash Sadrayi

arash.sadrayi@modares.ac.ir

Amin Mirza Boroujerdian

Boroujerdian@modares.ac.ir

1 Jalal, Iran 